\title{
Precision CNC machining and ways to achieve it
}

\author{
Roman Nekrasov ${ }^{1, *}$, Ulyana Putilova ${ }^{1}$, and Yulia Tempel $^{1}$ \\ ${ }^{1}$ FSBEI of Higher Education Tyumen Industrial University, Tyumen, Russian Federation, 625000
}

\begin{abstract}
The quality of the part is characterized by precision of its processing. A good fit of the product parts and, as a consequence, its reliability in general depends on how accurately the size and shape of the part is maintained during machining. In this connection, the article considers the problem of ensuring the accuracy during machining of work pieces using $\mathrm{CNC}$ machines. Within the identified problem, the analysis of known technical solutions in this area was carried out and three main areas were identified: optimization of cutting conditions, improvement of the treated surface area according to the criteria for increasing the resistance of the cutting tool, as well as error tracking and correction during machining. The main disadvantages of the analyzed methods and inventions are complexity of their implementation, the need to equip the $\mathrm{CNC}$ machine with an adaptive control system, and a limited field of application. Therefore, a method for controlling the accuracy of shaft processing using mathematical and finite element modeling is proposed.
\end{abstract}

Increasing the productivity and accuracy of metalworking operations, the quality of products is one of the topical issues in engineering technology and cutting theory. Machine equipment equipped with $\mathrm{CNC}$ systems has a high discreteness of movements, functions of adjusting the movement of the tool, accounting for wear of the cutting edge, etc. However, despite all the technical capabilities, the accuracy of processing does not always correspond to the preset one.

The problems of the cutting process optimization, increasing the machining accuracy and the quality of manufactured machine-building products were dealt with by such scientists as Yu.A. Rosenberg, T.N. Loladze, A.D. Makarov, N.N. Zorev, A.S. Verecschaka, Ye.V. Artamonov, M.Kh. Uteshev, Yu.I. Nekrasov, V.I. Zhiganov, L.K. Generalov, A.A. Matalin, V.E. Push, D.N. Reshetov, R. Pigert and many others.

Their works $[1,2,3,4,5]$ are connected with the increase of stability and efficiency of the cutting tool, optimization of the cutting modes, error estimation and compensation in the metalworking process, increasing the rigidity of the technological system and balancing, investigating the influence of the technological equipment nodes on the processing accuracy etc.

In the processing of metals by cutting, the most common are two directions of precision control. The first is associated with ensuring high accuracy of manufacturing and assembly of individual elements of the technological system, increasing its rigidity and reliability in

\footnotetext{
* Corresponding author: nekrasovri@,tyuiu.ru
} 
operation. The preset accuracy of processing is achieved due to the reduction of position errors and the movement of the actuating elements of the process equipment and cutting tools. The second direction is in optimization of the factors controlling the quality indicators of the technological process, namely, in the selection and designation of cutting modes and geometry of the cutting part of the cutting tool, selection of a rational grade of tool material, etc. The disadvantage of the second direction is uncertainty associated with a large range of technological factors. Along with these directions, a new application is found that is related to the development of automated control systems for the accuracy of machining [6,7].

The accuracy of processing is the most important characteristic of any technological equipment, in the work under consideration - a CNC machine tool. Under the accuracy of processing we understand the degree of conformity of the parameters for the manufactured part with nominal values. As you know, increasing the accuracy of manufacturing parts increases the service life of machines and equipment [2].

There is a number of works $[1,2,3,8,9,10]$, devoted to the problem of increasing the accuracy of machining on CNC machines. This problem was considered by such scientists in the field of technology of machine building and metal-cutting machines, as A.A. Matalin, V.E. Push, D.N. Reshetov, V.T. Portman, V.A. Ratmirov, Yu.V. Maksimov, A.A. Bekaev, V.V. Yurkevich, E.V. Leun, K.V. Khrustitskiy, A.A. Kutin, V.P. Legaev, A.F. Lysenko and others. The work of these scientists laid the foundation for further research into ways to improve the accuracy of machining on CNC machines.

Also the work of Yu.V. Petrakov belongs to the field of control of the cutting process on $\mathrm{CNC}$ machines, and in this research he presents a new method for controlling the cutting process when machining parts on CNC machines through known CAM-systems [11]. It should be noted that the design of control programs using standard cycles is widely used by manufacturers of CNC machines and is represented in the instructions of the operatorprogrammer. Such an approach, unfortunately, does not provide for the possibility of controlling the cutting regime [12].

In addition to the above methods of improving the accuracy of metalworking by taking into account errors and their correction, various inventions and utility models are patented in the field of active control of linear dimensions during processing, automatic control of the precision of billet machining, adaptive machining of products on $\mathrm{CNC}$ machines, control of geometric accuracy and adjustment of power parameters in the process of processing, software correction of executive movements by the machine tool.

For example, in patent No. 2379169 , V.S. Titov presented a method and a device for controlling the accuracy of machining of parts, the technical result of which is an increase in the geometric accuracy of the surface of parts machined on CNC machines by automated correction of the cutting and feeding speed, and the expansion of the functionality of the device [13].

From the above analysis of patent and literature sources, the following conclusions can be drawn. A significant problem today for modern machine-building enterprises is the accuracy and quality of the parts produced when designing programs for processing blanks and operating machine tools with numerical program control. In this regard, many researchers and scholars in this field seek to solve this problem in various ways, but pursue the same goal - the output of a high probability of products that meet the established requirements, as well as preservation of material resources, energy, labor and time when producing precision parts of modern production.

Among the significant directions in the research of authors in the field of increasing the accuracy and quality of processing are software correction of machine movements, development and research of intelligent control systems, automatic control of geometric accuracy and regulation of power parameters during processing. But, since a lot of factors 
influence the processing process, the actual task for today is to replenish the already existing knowledge base in the area under consideration and to increase the efficiency and effectiveness of modern machine-building production.

The disadvantages of the known technical solutions are a limited field of application, the need to equip the $\mathrm{CNC}$ machine with an adaptive control system, as well as the complexity of the proposed device design and the implementation of ways to improve the accuracy of metalworking.

In connection with the foregoing, we propose a method for controlling geometric accuracy, which consists in numerical and mathematical modeling of the shaping of a surface of a shaft-type part under the influence of elastic force deformations on an object of work, and automatically making changes to the initial geometric model recorded in the STEP format.

To determine the specific values of the spatial deviations of the real part profile from the nominal during the processing under the action of elastic force deformations, a simulation was carried out. The object of the study was a shaft of steel grade - Steel 40X (GOST 4543-71). As an example, we consider a work piece at turning, fixed in a lathe chuck without a back center.

For finite element analysis, having calculated the cutting force components arising during the turning of a cylindrical surface of a piece with a diameter of $33 \mathrm{~mm}$ and a length of $120 \mathrm{~mm}$ for a single rough turning, with a cutting depth $\mathrm{t}=2.5 \mathrm{~mm}$, a cutting tool T15K6 GOST $18878-73$ with the size of the holder $16 \times 25$ and the following geometric elements of the tool: the main angle in the plan $\varphi^{\circ}=45^{\circ}$; front angle $\gamma^{\circ}=0^{\circ}$; angle of inclination of the main blade $\lambda^{\circ}=0^{\circ}$, a calculation model with physic-mechanical properties of the selected material was created. The properties of the selected material are as follows: modulus of elasticity $\mathrm{E}=1.76 \cdot 10^{-5} \mathrm{MPa}$; Poisson's ratio $v=0.25$; density $\rho=7670 \mathrm{~kg}$ / $\mathrm{m} 3$. The load to be set is the components of the cutting force $\mathrm{P} z, \mathrm{y}, \mathrm{x}=2798 / 1392 / 1392 \mathrm{~N}$.

The results of the finite element analysis showed a change in the spatial shape of the object under study, the magnitude of the resulting displacements of which is shown in Figure 1, they are indicated in micrometers.

In connection with the above, based on the numerical simulation, the next step was the introduction of changes to the original geometric model recorded in the STEP format.

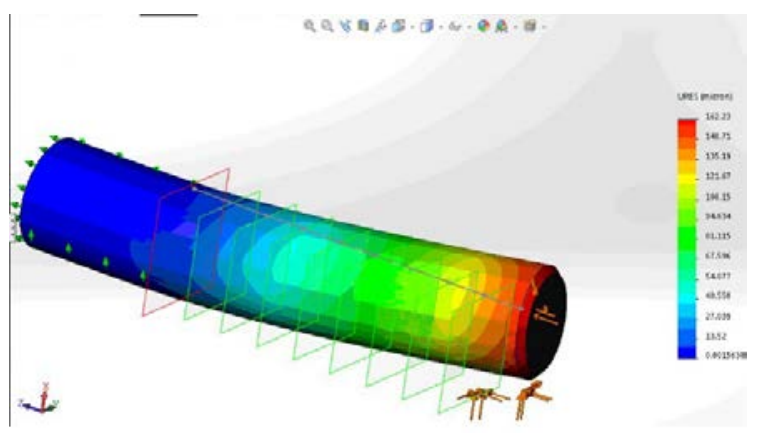

Fig. 1. The results of analysis of the finite element model movement

To confirm the effectiveness of the proposed method for controlling the geometric accuracy of the work piece on CNC machines, two batches of parts were machined on the turning center SMTCL CAK50135 with a straight cutting tool T15K6 GOST 18882-73 with a holder size of $16 \times 25$. The first batch was processed without taking into account the arising errors, the second batch of parts was to be processed according to the program with the modified trajectory of the tool tip movement on the basis of the transformed CADmodel. The results of the experiments are shown in Fig. 2 

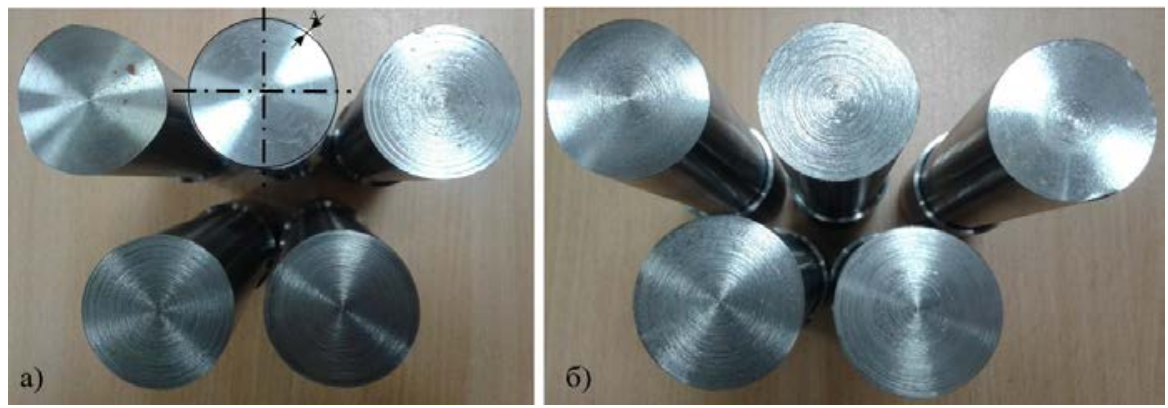

Fig. 2. Surfaces of the treated shaft: a - without changing the trajectory of the machine control elements; $\sigma$ - taking into account the change in the trajectory of the machine control elements, according to the proposed method for controlling the geometric accuracy of the machining

To evaluate the reliability indexes on the quality parameters of the manufactured products, depending on the type of technical system and the purpose of the assessment, experimental-statistical methods were used.

To determine the probability percentage of spoilage when manufacturing a batch of parts within the tolerance for size, a Gaussian distribution curve was constructed from the data of the same diametral dimension di for the first and second batches of parts, as shown in Figure 3 (No. 1 and No. 2 respectively). In Figure 3 the following designations are assumed: F1 is the area determining percentage of repairable spoilage; F2 is the area that determines the percentage of suitable parts in the batch (within the tolerance range); F3 is the area that determines the percentage of incorrigible spoilage; $\omega_{\mathrm{di}}$ is the scattering field; TA - tolerance field; $\bar{d}_{i}$ - arithmetic average value of the actual shaft size; $\mathrm{S}_{\mathrm{d}}$ is the standard deviation.

When comparing the results of measurements and determining reliability of the technological operation when processing the parts without changing the control program and in accordance with the proposed control method of the geometrical accuracy of processing, it can be said that the efficiency and effectiveness of the method is confirmed by experiment and analysis of the data obtained in the experiment. Since the analysis of the distribution of a random variable shows a constant bias in the batch of parts processed by the control program with a transformable CAD model, in addition, the standard deviation is reduced by $40.9 \%$, and the range of variation is reduced almost twofold.

Thus, the realization of the research achieved the following goal: increasing the dimensional accuracy of the formation of parts such as bodies of revolution when turning on machines with numerical program control.

Reliability of the obtained results is confirmed by numerical and physical experiments. Assessment of the reliability of the technological operation of machining two batches of parts and comparing the results allows us to make a conclusion about effectiveness of the proposed method for controlling the geometrical accuracy of machining on CNC machines. 


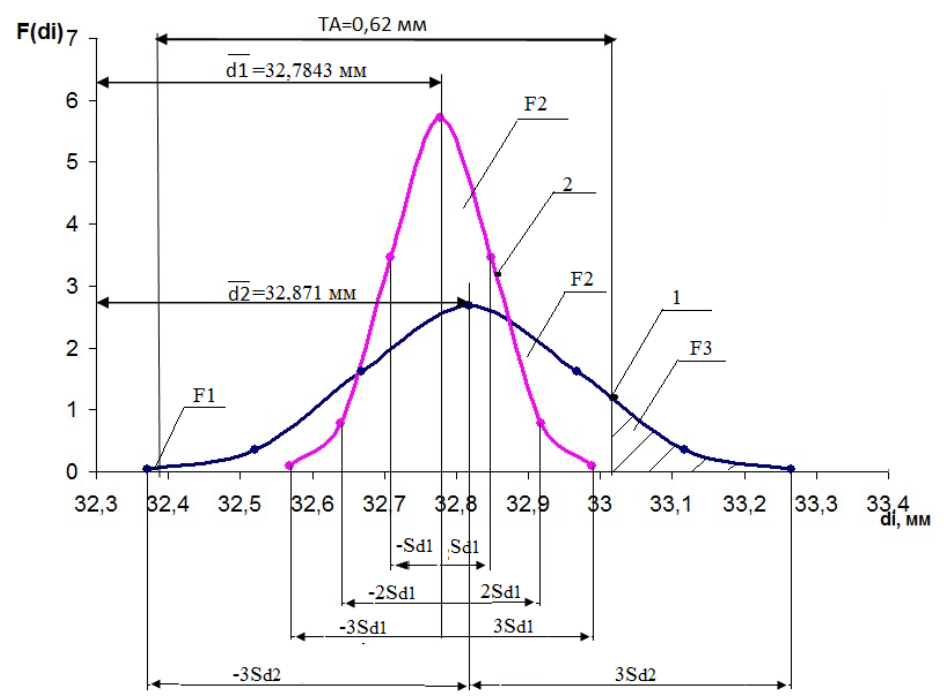

Fig. 3. Differential distribution functions of a random variable when measuring parts processed in accordance with the proposed method for controlling geometric accuracy - 1 and processed in accordance with the traditional method of machining - 2

When comparing the results of measurements and determining reliability of the technological operation when processing the parts without changing the control program and when processing the parts according to the proposed control method of geometrical accuracy of processing, it can be said that efficiency and effectiveness of the method was confirmed by the experiment and analysis of the data obtained in the experiment. Since the analysis of the distribution of a random variable shows a constant bias in the batch of parts processed by the control program with a transformable CAD model, in addition, the standard deviation was reduced by $40.9 \%$, and the range of variation was reduced almost twofold.

Thus, the realization of the research achieved the following goal: increasing the dimensional accuracy of the formation of parts such as bodies of revolution when turning on machines with numerical program control.

Reliability of the obtained results is confirmed by numerical and physical experiments. Assessment of the reliability of the technological operation of machining two batches of parts and comparing the results allows us to make a conclusion about effectiveness of the proposed method for controlling the geometrical accuracy of machining on CNC machines.

\section{References}

1. U.S. Putilova, Y.I. Nekrasov, A.A. Lasukov, A.A., Applied Mechanics and Materials, Loading of the manufacturing systems elements in the process of unsteady mode cutting and the models of their arrangement deviations, 682, pp. 192-195 (2014).

2. R.Y. Nekrasov, I.V.. Solovyov, I.V., A.I. Starikov, O.V. Bekareva, Key Engineering Materials, Improving dimensional accuracy of turning on CNC equipment, 736, pp. 95100 (2017).

3. R.Y. Nekrasov, I.V.. Solovyov, I.V., A.I. Starikov, Y.A. Tempel, O.A. Tempel, MATEC Web of Conferences, Numerical studies to determine spatial deviations of a workpiece that occur when machining on CNC machines, 129 (2017). 
4. E.V. Artamonov, M.O. Chernyshov, T.E. Pomigalova, Russian Engineering Research, Improving the performance of modular drills with interchangeable cutting heads, 37(7), pp. 626-627 (2017).

5. E.V. Artamonov, D.V. Vasil'ev, V.V. Kireev, M.K. Uteshev, Russian Engineering Research, Mechanics of chip formation in cutting, 37(7), pp. 450-454 (2017).

6. E.A. Kudryashov, I.M. Smirnov, Manufacturing Technology, Tool guarantee of intermittent cutting processes, 17(6), pp. 887-892 (2017).

7. E.A. Kudryashov, E.V. Pavlov, I.M. Smirnov, Journal of Mining Science, Justification of Damping Cutter Design for Making Parts for High-Pressure Drilling Unit Cylinders, 53(3), pp. 484-488 (2017).

8. I.A. Kovalev, P.A. Nikishechkin, A.S. Grigoriev, International Conference on Industrial Engineering, Applications and Manufacturing, ICIEAM 2017 - Proceedings, Approach to programmable controller building by its main modules synthesizing based on requirements specification for industrial automation (2017).

9. G.M. Martinov, N.V. Kozak, R.A. Nezhmetdinov, A.I. Obukhov, L.I. Martinova, Automation and Remote Control, Method of decomposition and synthesis of the custom CNC systems, 78(3), pp. 525-536 (2017).

10. G.M. Martinov, S.V. Sokolov, L.I. Martinova, A.S. Grigoryev, P.A. Nikishechkin, Lecture Notes in Computer Science (including subseries Lecture Notes in Artificial Intelligence and Lecture Notes in Bioinformatics), 10386 LNCS, pp. 586-594 (2017).

11. M. Raoufinia, Y.V. Petrakov, A. Ataei, R. Parand, K. Abou-El-Hossein, Journal of Applied Sciences, Error compensation of complex three-dimensional surfaces machined on computer-numeric-control grinding machine tools, 9(7), pp. 1356-1361 (2009).

12. Y. Petrakov, M. Danylchenko, A. Petryshyn, EasternEuropean Journal of Enterprise Technologies, Programming spindle speed variation in turning, 2(1-85), pp. 4-9 (2017).

13. Patent № 2379169, MPK B23Q15/00, publ. 20.01.2010. 